\title{
Kinematic Mapping of the Narrow Line Region of NGC4151 ${ }^{1}$
}

\author{
M.E. Kaiser ${ }^{2}$, L.D. Bradley II
}

Dept of Physics and Astronomy, Johns Hopkins University, Baltimore, MD 21218

\section{J.B. Hutchings ${ }^{2}$}

Dominion Astrophysical Observatory, National Research Council of Canada, 5071 W. Saanich Rd., Victoria B.C. V8X 4M6, Canada

S.B.Kraemer ${ }^{2}$, D.M. Crenshaw, J. Ruiz

Dept of Physics, Catholic University of America, Washington DC 20064

D. Weistrop ${ }^{2}$, C. Nelson

Dept of Physics, University of Nevada, Las Vegas, 4505 Maryland Pkwy, Las Vegas, NV 89154-4002

T.R. Gull ${ }^{2}$

NASA Goddard Space Flight Center,Lab for Astronomy and Solar Physics, Code 681, Greenbelt MD 20771

We present HST Space Telescope Imaging Spectrograph (STIS) slitless spectroscopy of the NGC4151 narrow line region (NLR) as a probe of the kinematic stucture of the extended emission-line gas emanating from the nucleus. Using slitless spectroscopy at two roll angles (with a spatial resolution of $0.051^{\prime \prime} /$ pixel and a point source spectral resolution of $0.55 \AA$ ) augmented with narrow band images, we have mapped the velocity field of the NLR as defined by $\sim 60$ discrete cloud structures in [OIII]. Flux measurements of [OII], $\mathrm{H} \beta$, [OIII], [OI], and [SII] were made for individual cloud structures wherever possible.

The emission line clouds have a biconical distribution which extends further SW than NE of the nucleus (Figure 1). In general, the SW clouds have approaching velocities, whereas the NE clouds are receding. Higher velocity clouds, as well as higher velocity dispersion clouds, are located within approximately $\pm 180 \mathrm{pc}$ of the nucleus. The bicone axis is roughly coincident with the radio axis (Pedlar et al., 1993, Winge et al., 1997).

\footnotetext{
${ }^{1}$ Based on observations with the NASA/ESA Hubble Space Telescope, obtained at the Space Telescope Science Institute, which is operated by AURA Inc under NASA contract NAS526555

${ }^{2}$ Co-investigator STIS Instrument Defininition Team, funded in response to NASA Announcement of Opportunity OSSA-4-84
} 


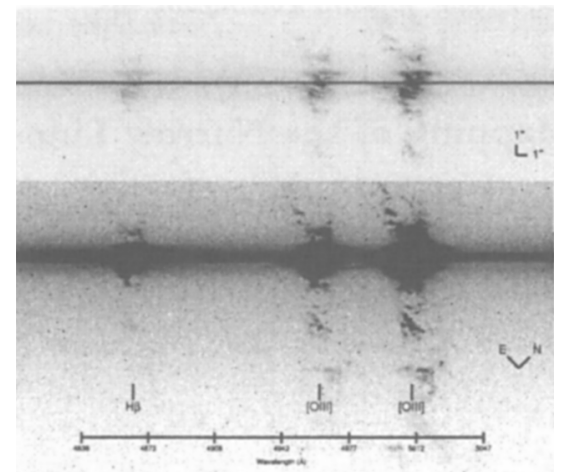

Figure 1. Slitless spectrum of NGC4151, illustrating the capability of this mode to spatially map the biconical emission line structure while simultaneously providing a kinematic map of the $\mathrm{H} \beta$ and [OIII] clouds.

Our velocity measurements indicate that there are two distinct kinematic components. One component is characterized by lower velocities $\left(\left|v_{\text {rel }}\right|<400\right.$ $k m s^{-1}$ ). The other population of clouds is characterized by both high velocities and high velocity dispersions $\left(400<\left|v_{\text {rel }}\right|<1700 \mathrm{~km} \mathrm{~s}^{-1}, \sigma_{V} \gtrsim 130 \mathrm{~km} \mathrm{~s}^{-1}\right)$.

We have modelled the velocity distribution by simple Keplerian rotation about a central source. At large radii the gas kinematics are consistent with normal galaxy rotation and have a low amplitude since NGC4151 is quite faceon. We find a rather poor fit to both the high and low velocity clouds closer to the nucleus, effectively eliminating rotation around a black hole as the origin for the gas motions.

For the high velocity cloud population another physical mechanism, such as wind driven outflow, must be responsible for the gas kinematics. We argue against infall based upon opacity and similarity arguments. (1) The blueshifted clouds are brighter and have a more extended distribution. Since we expect clouds on the near side of the nucleus to appear brighter, this implies outflow. (2) The CIV absorbers all have outflow velocities (Hutchings et al. 1998, Weymann et al. 1997). (3) Outflow is consistent with the radio observations (Ulvestad et al., 1998, Pedlar et al. 1993).

Several of the clouds lie near, but not coincident with, the radio axis. However, we have found no strong correlation between either velocity or velocity dispersion and proximity to the radio cores.

Flux measurements of the individual clouds are consistent with photoionization. The $[\mathrm{OIII}] / \mathrm{H} \beta$ ratio declines roughly linearly within an inner $\sim 3^{\prime \prime}(200 \mathrm{pc})$ radius from the nucleus. This decrease in the flux ratio indicates a decreasing ionization parameter (Ferland and Netzer, 1983). Since the ionization parameter is proportional to $r^{-2} n^{-1}$, it appears that the density, $n$, must decrease as $\sim r^{-1}$. At distances further from the nucleus, the $[\mathrm{OIII}] / \mathrm{H} \beta$ ratio is roughly constant and comparable to its initial value, indicating that either the ionization parameter is roughly constant at larger radii with the density decreasing as $r^{-2}$ or we are subject to projection effects, local gradients, or both. 


\section{References}

Ferland, G.J., and Netzer, H., Ap.J., , 264, 105

Hutchings, J.B., Crenshaw, D.M., Kaiser, M.E., Kraemer, S.B., Weistrop, D., Baum, S., Bowers, C.B., Feinberg, L. D., Green, R.F., Gull, T.R., Hartig, G.F., Hill, G., Lindler, D.J., 1998, ApJ, 492, L115

Pedlar, A., Kukula, M.J., Longley, D.P.T., Muxlow, T.,W.B., Axon, D.J., Baum, S., O’Dea, C., Unger, S.W. 1993, M.N.R.A.S., , 263, 471

Ulvestad J.S., Roy, A.L., Colbert, E.J.M., Wilson, A.S., 1998, Ap.J., , 496, 196

Weymann, R.J., Morris, S.L., Gray, M.E., Hutchings, J.B., Ap.J., , 483, 717

Winge, C., Axon, D.J., Macchetto, F.D., Capetti, A., 1997, ApJ, 487, L121 